\title{
DIRECT OBSERVATION: IMPEDIMENTS AND APPROACHES
}

\author{
John Richer \\ Paediatric Psychology, Oxford University Hospitals and Department of Physiology, \\ Anatomy and Genetics, University of Oxford \\ johnricher@oxhs.co.uk
}

\begin{abstract}
This paper explores why direct observation of our own species behaviour is so little done in psychology and other social sciences. The very familiarity and the richness of our cultural understanding of our behaviour is paradoxically an impediment, since that understanding is couched in the language of an active participant in social interactions. That language acknowledges and communicates the subjective states of ourselves and others but it is not couched in the language of a scientist, who is an outsider, a passive observer, who does not use the terms of his subjects and does not study their subjectivity since it is not publically observable and cannot be agreed upon in the usual scientific way of demonstrating a phenomenon for anyone to observe. A major function of our rich everyday knowledge is to help us be successful social participants. That type of practical knowledge is not necessarily useful for a detached science.

Other impediments include

- $\quad$ our moral distaste at viewing our fellow human beings as objects without subjectivity

- initial observations can seem banal and uninformatively familiar

- direct observation is time consuming and expensive

- $\quad$ specific research hypotheses seem lacking
\end{abstract}

Two traditional ethological ways of describing behaviours - by morphology and by consequence. A disciplined seriously playful approach is advocated for the early stages of research, trying to find useful behaviour categories to measure. One aid to this is to look for natural divisions in the phenomena studied and quotes the Plooij's work comparing children's behaviour either side of regression (rapid development) periods in early development. 
Keywords: Direct observation, knowledge and agreement, minds, natural environments, disciplined playfulness, developmental discontinuities

When Bill Charlesworth, one of the founders of ISHE, urged scientists of human behaviour to "follow the duck not the theory of the duck", he was, in his characteristically humorous and cryptic way, urging people to look at naturally occurring behaviour as the starting point of their investigations, rather than what people thought ducks (a.k.a. people) were doing, their theory of the duck behaviour. It seems obvious that any science starts with asking, "what are the natural phenomena to be explained?". So why did this apparently obvious and incontravertable remark need to be made?

Whilst there is much psychology that has made a great contribution to knowledge and to the practical benefit of people, too much seems just not founded on a solid basis of observation of human behaviour, but instead on plausible, but culturally founded, ways of understanding human behaviour and thought. The theory of the duck has been followed, not the duck.

But why is this a problem for the scientific study of human behaviour, and, connectedly, why it is that doing direct observation seems so difficult?

\section{IMPEDIMENTS TO DIRECT OBSERVATION}

\subsection{We know it already}

A major impediment is that we think it is unnecessary, since we already know about human behaviour. As sophisticated social beings dealing fairly successfully with each other daily, we have a lot of knowledge about what to expect from others. This extensive rich understanding of people is seen in many areas, including:

- everyday language and culture,

- proverbs, legends, sagas

- literature, drama, dance, art, music

- philosophies, religions

- law, politics, journalism

But this is not a single body of knowledge, it is culture specific (although with large similarities, unsurprisingly, between cultures). By contrast science aspires to a single body of knowledge, although many fields, especially in the social sciences, have competing paradigms: the bodies of theory, methodology, accepted facts etc. that characterise each paradigm, and those paradigms shift, often suddenly, over time (Kuhn, 1962).

This knowledge about people's behaviour is not based on well defined and documented, countable categories of publically observable behaviour. If it was, our advocacy for direct observation would be redundant! 


\subsection{Two types of knowledge}

More crucially, this cultural knowledge has a different function: it helps us cooperate and compete with each other, it helps us understand our social and physical world sufficiently to be able to operate successfully within it. It is a practical knowledge. More than that we do things with words (Austin, 1960), not just, for instance, the "I do" in the English marriage ceremony, but also, say, in describing someone in a certain way we may hope to influence others to have that view. The ideas we have about people and the way we use those ideas are an integral part of our functioning within our culture.

This leads to the argument, which I have made before (Richer, 1975, 2016) that there are two types of knowledge about human behaviour. One is the knowledge familiar to everyone. It is embodied in our cultures, languages and everyday dealings with each. It the language of the active participant, the agent. It comes naturally to us.

The other is the language of science, whose forerunners were the objective observations that people made about each other and their worlds. Here the stance was of the dispassionate detached observer, the onlooker. Concepts associated with these two types of knowledge are summarised in this table (from Richer, 2016).

Table 1. The two types of knowledge about human behaviour

\begin{tabular}{ll}
\multicolumn{1}{c}{ Agent } & \multicolumn{1}{c}{ Onlooker } \\
\hline Sharing Minds & Observing Bodies \\
Agency involved, action & Onlooking only, events \\
I do & He/she/it does, or, It happens \\
Feelings, intentions, reasons & Causes, effects, goals \\
Free will & Determinism \\
Emic (culture's own terms) & Etic (observer's terms) \\
Useful for communication in a culture & For communication about a culture \\
Arts, Religions & Sciences \\
Persuasion & Manipulation \\
Symptoms & Signs \\
Content of consciousness & Phenomenon of intersubjectivity \\
Demonstrated agreements not required & Demonstrated agreements required \\
\hline
\end{tabular}

\subsection{Two types of knowledge, two types of agreement}

These two types of knowledge are, to state the obvious, shared. If they were not we could not talk about them It is possible for an individual to have considerable (private) knowledge, wisdom even, but it not be shared, but then it dies when that individual dies. Given that the knowledge we can discuss is shared then the crucial question is not how an individual comes to know something, but how individuals can come to agree. 
Two types of agreements may be distinguished: Negotiated ( $\mathrm{N}$ type) and Demonstrated (D type) (Richer, 1975). An example of Negotiated agreements is: we agree that this colour means "red", or that "flower" means this sort of thing and we agree on the use of "this" and "is". What words (in the sense of the sounds we make or the marks on paper) are used is largely arbitrary, (and different in different languages) and the exact boundaries of the meanings are sometimes fuzzy and/or different in different languages (Nagel, 2014).

Possessing these Negotiated agreements enables us to proceed to Demonstrated agreements. A Demonstrated agreement is of the type, for example, "this flower is red". Having agreed the meanings of each word we can agree, or disagree, on "this flower is red" simply by demonstration, perhaps by pointing at it.

Negotiated agreements can be changed and are judged by whether they are useful, demonstrated agreements are judged by whether they describe the real shared world accurately, by whether they are, in this sense, true.

\begin{tabular}{ll}
\hline \multicolumn{1}{c}{ Type of agreement } & \multicolumn{1}{c}{ Evaluated according to } \\
\hline N Negotiated & Usefulness \\
D Demonstrated & Truth \\
\hline
\end{tabular}

\subsection{Agreements in Science}

In science this agreement process is formalised and tightened: terms are carefully and precisely defined, or at least the attempt is made to do so, and the phenomena under study are described in these terms. A hypothesis couched in these terms can be tested by an attempt at demonstration, such that, having accepted the terms, the scientists can agree, after a demonstration, on what the result is. Science, as Medawar (1967) argued, is about creating a coherent story about the facts and which can be tested by seeing whether it accords with the facts. The facts are the demonstrated agreements.

The actual conduct of science is not so neat as this. Methodology and terminology are disputed. There can be debate, (negotiation), about the meanings of terms. This is often not a dispassionate process, a lot of politics and persuasion enters into it. The persuasive powerful professor will often get his definition agreed even though many may privately hold the view that it is less useful than its competitors. Lehrman (1970) argued that disputes in science are usually about the terms used and rarely about the facts.

\subsection{Agreements in Direct Observation}

The obvious needs stating briefly. The definition of a behaviour category is agreed essentially by negotiation. Then data like the frequency, occurrence in different setting and relationship to other variables, sequences, etc., can be agreed by demonstration. 


\subsection{Unnatural, alienating and amoral?}

I have already argued that there are two stories to be told about human behaviour. One is the everyday one which comes naturally to us all, it involves agency, subjectivity and contains implicit values, not least is a respect for the subjectivity and intrinsic value of the other person.

Direct observation cannot embrace the subjectivity of the people observed since that cannot be agreed by demonstration, only negotiation with that person (Richer 1975, 2016). So, in direct observation, the other person is seen as a biological machine, rather than an moral agent with free will. As such their moral value seems equivalent to that of an ant or a tree. This seems alienating and amoral, it seems like we are adopting the mentality of psychopaths, genocidal killers and the like, and most of us have a strong aversion to thinking like that.

Yet it can be done without sacrificing moral respect. It is, for instance, what clinicians do daily, looking at signs of illnesses (objective) and asking about symptoms (the complaints which the patient describes), and do this with the wishes and the welfare of the patient as the central consideration.

\subsection{The behaviour is too familiar}

Whilst it may seem an advantage to be familiar with the behaviour observed, in fact it often inhibits good observation. Too often the familiarity means that details are missed, and it is difficult not to see the behaviour in the ordinary terms of the observer's culture, which are not defined and which usually carry implications of subjectivity, agency and morality.

Direct observation is easier when the behaviour seems odd and is not so amenable to everyday description. This is the case observing the disordered behaviour of those with psychiatric diagnoses, or observing babies or people from very different cultures.

Some researchers try and make behaviour unfamiliar by recording behaviour then speeding it up or slowing it down, or in some other way making it unusual.

\subsection{Less useful, more time consuming and expensive}

Direct observation takes time and therefore money, and many funding organisations are unwilling to support this work. This is especially true when the results seem to many to be less useful than everyday observations.

\subsection{Apparent lack of specific research hypotheses}

Many funding organisations require clear research questions and tend not to encourage apparently open ended research. There are good reasons for this, not least of which is that one way in which science advances is by testing clear hypotheses. Without clarity and without data which demonstrably supports a hypothesis or not, little advance is achieved.

But one can have clear questions without having specific hypotheses. Direct observation is not idle observation, or creating descriptions with no aims. At its most 
basic, direct observation is what Tinbergen called "watching and wondering". The wondering "why does that animal do that?" was clarified into four key questions, (Tinbergen's "4 why?":) immediate causation, ontogeny, function and phylogeny. These are questions which any observer has in their mind. (Tinbergen, 1963). They provide an invaluable framework to guide observation.

\subsection{Direct Observation is biassed}

Some have argued that direct observation is always biased by the observer's preconceptions (e.g. Cooper et al, 1974, reply by Richer, 1974), and thus even this basic data set of the science is distorted. But this position makes at least two mistakes. The first is that of course there are preconceptions, but the observer tries to be aware of them and minimise them. The second is that, as just stated, the observer brings the ethological framework of Tinbergen's " 4 Whys?", to guide the direct observation, if that counts as a "preconception" or "bias", then it is a happy and heuristic one.

\subsection{Most environments are not "natural"}

Direct observation in the "natural" environment is occasionally argued to be virtually impossible since modern environments are so far removed from what is thought to be our environment of evolutionary adaptedness, namely that of the Hunter Gatherer. But there are two errors in this argument.

The first is that the choice of the Hunter Gatherer environment and lifestyle is arbitrary. Why not periods before or after?

The second is to misunderstand the use of the word "natural" in this context. "Natural" can refer to any environment people are in and where they are observed. The term "natural" simply means minimally affected by the observer. A riposte to this has been that the observer can never say he does not affect what is observed, which is true, but the observer tries to minimise that influence and always bear in mind that there might be some influence.

To summarise: Direct Observation is

- open minded observation, guided mainly only, at least at first, by the general biological questions of the " 4 Whys"

- $\quad$ of publically observable behaviour,(i.e. what can be agreed by Demonstration)

- which occurs naturally (i.e. with minimal researcher intervention)

\section{FIRST STAGES OF OBSERVATION}

I am indebted to Frans Plooij for the following quote from Schneirla (1950).

"It is necessary intellectually to soak in the environmental complex of the animal to be studied until we have a facility with it which keeps us as it were, one move ahead”. From there onwards there is a progression from direct observation in the field to 'field experiments' to experiments in the lab. 
Scientists who have spent a considerable time directly observing a set of individuals will recognise this sense that one can frequently intuitively predict what an animal / person is about to do, even if one cannot be explicit about it.

To become explicit, and create behaviour categories, the initial approach can draw on this intuition. It can be playful, in the serious sense of trying out different categories to see if they seem to yield interesting observations. Behaviour categories, it needs to be remembered, like other negotiated agreements, are judged by their usefulness, in this case their scientific usefulness in furthering understanding of the natural phenomena.

Part of this is that they have to capable of being used reliably by different observers, and so, for instance, inter- and intra-relability studies are done.

\section{TYPES OF BEHAVIOUR CATEGORIES}

Long ago, Hinde (1970) summarised the two main ways of defining a behaviour category, namely by:

- Morphology

- Consequences

Some examples:

Morphology: Smile, point, fingers in the ears, hands covering the eyes, eat, fear grin.

Consequences: approach, escape, avoid, build a tower, follow, upset another.

A major issue is whether or not to lump together similar seeming behaviours. Lumping together can simplify data collection and analysis and may yield patterns which get lost in the complex noise of too many categories. But equally it may obscure important discriminations and so important phenomena are missed. The general rule in ethology is "split, don't lump", for the very simple reason that smaller categories may be lumped together at the stage of data analysis, but larger categories cannot be split.

This process can be greatly aided by looking for natural dividers, and asking what seems to differ between two sides of a clear division.

Age is one example, but not using the arbitary units of months and years, instead looking for real discontinuities in development and then asking what has changed comparing behaviour before and after. This was the approach of the Plooijs in their brilliant plotting of early developmental changes (van de Rijt Plooij and Plooij, 1992, 1993; Plooij and van der Rijt Plooij, 1988; Plooij, 2003). They had previously looked at the development of infant chimpanzees and applied a similar approach to human infants. They noted a number of periods where that infants were more difficult. These were called "regression periods" because the baby's behaviour regressed (they became "crying, cranky, and clingy" as they later described it in popular parenting books). They found these occured at predictable weeks of age (measured from due date). Most of the 10 such periods in the first 18 months, coincided with spurts in brain browth. They noted that the usual developmental milestones such as sitting, crawling, walking, talking etc., were very variable in their age of onset. So instead they looked at what the baby could understand before and after and found they had made a developmental leap. What was rapidly developing during each regression period was the input complexity that the baby 
could process, and which would thus define the goals which guided behaviour. They placed their work within Perceptual Control Theory of Powers (1973) which emphasises the way in which animals behaviour to achieve certain inputs (so they can only strive to achieve what they can "perceive" / process). They also described a hierarchy of input analysis in which lower analyses combine to enable a higher order analysis. The Plooijs plot this developmentally showing the age at which the child is capable of each analysis. Thus patterns are revealed in the development of children's behaviour not by starting from arbitary choices of behaviours (e.g. reaching, walking, talking, or worse, intelligence, perception, etc.) but by asking where, to put it perhaps too lyrically, nature places discontinuities. Then they ask how behaviour is different either side of those discontinuities.

Another example is taken from my own work with children diagnosed as autistic. Before the 1980s these children were said to be unaware of others, to be insensitive to pain, to laugh inappropriately etc. (DSMIII). Direct observation showed these assertions to be incorrect.

Unawareness of people. It revealed regular patterns of reaction to others (the children showed a predominance of avoidance behaviour over sociable behaviour) (Richer 1976).

Insensitivity to pain. The children clearly reacted to potentially or actually injurious events but they often did not cry or seek comfort. When they inflicted injury on themselves it was a times when their other behaviour indicated great stress and this self injury was an extreme displacement activity much like the self harming of many adolescent girls (whom nobody accuses of being insensitive to pain).

Inappropriate laughing / smiling Close observation of this "smiling" and "laughing" showed that the shape of the mouth and other features in the face were different from greeting or playful smiles. In particular the upper lip was straighter than in a smile and different sets of muscles seemed to be being used. The eyebrows were either lowered protectively or were raised for a long time, which is sometimes associated with search for an escape route in flight (Blurton Jones,1974; Darwin, 1872) unlike the eybrow flash of greeting (Eibl-Eibesfeldt, 1972),. The laughing was monotonous, high pitched and often staccatto, (colloquially, it sounded hysterical). This behaviour occured in the same situations as other avoidance behaviour, and together with other avoidance behaviour. Thus the nature and meaning of this behaviour was entirely different from that of the smiling and laughing of most children.

These two examples also illustrate the point made earlier, that useful direct observation often comes more easily when babies or psychiatric groups are observed, both not fully integrated into their cultures: babies because their have not yet acquired their cultural skills, the psychiatric groups because their behaviour often distances them from the mainstream.

\section{REFERENCES}

Austin, J.L. (1979) Performative Utterances. In J.O. Urmson \& G.J. Warnock (Eds), Philosophical Papers. Oxford. OUP. DOI

Blurton Jones, N.G. \& Konner, M.J. (1970). An experiment on eyebrow-raising and visual searching in children. Journal of Child Psychology and Psychiatry, 11, 233-240. DOI 
Blurton Jones, N. (1974) Pers. Comm.

Cooper, E.S., Costello, A.J., Douglas, J.W., Ingelby, J.D., \& Turner, R.K. (1974). Direct Observation? Bullletin of the British Psychological Society, 27, 3-7.

Darwin, C. (1872). The Expressions of Emotions in Animals and Man. New York: D. Appleton \& Company. DOI

Eibl-Eibesfeldt, I. (1972). Similarities and differences between cultures in expressive movements. In R. A. Hinde, Non-verbal communication. Oxford, England: Cambridge U. Press.

Hinde, R.A. (1970). Animal Behaviour. New York. McGraw Hill

Kuhn, T. (1962). The Structure of Scientific Revolutions. Chicago: University of Chicago Press. DOI

Lehrman, D.S. (1970). Semantic and conceptual issues in the nature-nurture problem. In L. Aronsen, E. Tobach, D.S. Lehrman \& J.S. Rosenblatt (eds). Development and evolution of behavior (pp 17-52). New York, W.H.Freeman.

Medawar, P.B. (1967) Science, the Art of the Soluble. Methuen, London.

Nagel, J. (2014) Knowledge: A Very Short Introduction. Oxford, Oxford University Press. DOI

Plooij, F.X. \& van de Rijt-Plooij, H.H.C. (1988). Vulnerable periods during infancy. Hierarchically reorganized systems control, stress and disease. Ethology and Sociobiology, 10(4), 297-296. DOI

Plooij F.X. (2003). The Trilogy of Mind. In M., Heimann. Regression Periods in Human Infancy. London. Lawrence Erlbaum Associates.

Powers, W.T. (1973). Behaviour: the Control of Perception. Chicago, Aldine.

Richer, J.M. (1974). Direct Observation - a reply to Cooper et al.. Bulletin of the British Psychological Society, 27, 500-502.

Richer, J.M. (1975). Two types of agreement: two types of psychology. Bulletin of the British Psychological Society, 28, 342-345. (Reprinted in D., Child (ed.). Readings in psychology for the Teacher. Holt, Rinehart, and Winston, London 1977)

Richer, J.M. (1976). The Social avoidance in autistic children. Animal Behaviour, 24(4), 898-906. DOI

Richer, J.M. (2016). Mentalistic and scientific stories about human behavior, biomimetic heuristics and psychology's confusions. Human Ethology Bulletin, 31(4), 15-33. DOI

Schneirla, T. C. (1950). The relationship between observation and experimentation in the field study of behavior. Annals of the New York Academy of Sciences, 51(6), 1022-1044. DOI

Tinbergen, N. (1963). On the aims and methods of ethology. Zeitschrift für Tierpsychologie, 20(4), 410-33. DOI

van de Rijt-Plooij, H.H.C. \& Plooij, F.X. (1992). Infantile regressions: disorganization and the onset of transition periods. Journal of Reproductive and Infant Psychology, 10(3), 129-149. DOI

van de Rijt-Plooij, H.H.C. \& Plooij, F.X. (1993). Distinct periods of mother-infant conflict in normal development: sources of progress and germs of pathology. Journal of Child Psychology and Psychiatry, 34(2), 229-245. DOI 